\title{
Common genetic susceptibility to DCIS and invasive ductal carcinoma
}

\author{
Victoria Sopik and Steven A. Narod* \\ See related research by Petridis et al., http://breast-cancer-research.biomedcentral.com/articles/10.1186/s13058-016-0675-7
}

In a recent issue of Breast Cancer Research, Petridis and colleagues report a failure to detect any genetic polymorphism which predisposes to ductal carcinoma in situ (DCIS) but not to invasive ductal carcinoma (IDC) or vice versa [1]. They genotyped 5067 cases of DCIS, 24,584 cases of IDC, and 37,467 non-cancer controls for 76 different single nucleotide polymorphisms (SNPs), each of which has been shown to be associated with breast cancer susceptibility in previous studies $[2,3]$. They found no significant difference in the magnitude of the associations for DCIS and IDC for any of the 76 loci. From this, the authors conclude that the genes responsible for DCIS and IDC are, by and large, the same and that larger studies are required to determine if susceptibility loci specific to DCIS exist.

The authors base the rationale for this study on the premise that DCIS and IDC are distinct pathologies, in that DCIS is non-invasive and is a non-obligate 'precursor' lesion. Accordingly, they seek a biological basis which helps underpin the distinction. A simpler and parsimonious explanation for the overlap in gene sets is that DCIS and IDC represent various phases of the same disease process, rather than distinct forms of cancer, as we have recently proposed [4]. In this sense, it is unlikely that they will identify genes which predispose to stage 0 but not to stage I breast cancer (as one would not expect to find genes that predispose to stage II but not stage I cancer). If one wishes to study the possibility that host factors are associated with cancer stage at presentation-and with rate of progression-it is better to study breast cancers in their entire range (from stage 0 to stage IV) and to look for overall trends in prevalence.

\section{Authors' response}

\section{Christos Petridis, Sarah E. Pinder, lan Tomlinson, Rebecca Roylance and Elinor J. Sawyer}

In response to the comments by Sopik and Narod we would like to point out that the premise of our study was not that DCIS and IDC are distinct pathologies and we were not therefore seeking a biological basis to explain this distinction. Indeed we state that: " $45-78 \%$ of all invasive breast cancers are associated with DCIS and it is hypothesized in the majority of these cases that the invasive component has arisen from the DCIS as they generally share the same somatic genetic changes" [1]. We agree with the obvious explanation, which is that DCIS and IDC represent various phases of the same disease process.

\footnotetext{
* Correspondence: steven.narod@wchospital.ca Women's College Research Institute, 76 Grenville Street, Toronto, Ontario, Canada
}

Nevertheless, it is also clear that not all DCIS progresses to an invasive lesion. In particular, there are concerns regarding over-diagnosis and over-treatment of DCIS through screening programmes [5]. Current methods for accurately predicting the behaviour of an individual DCIS lesion are poor, with many researchers attempting to identify molecular biomarkers that can be used to distinguish between aggressive and nonaggressive DCIS with, to date, little success. The rationale for our study was to ascertain whether a subgroup of non-aggressive DCIS could be identified by examining low-risk genetic susceptibility loci, rather than looking at somatic changes within DCIS.

In our study we examined the extent to which DCIS without associated invasive disease (5067 cases) and IDC $(24,584$ cases $)$ share low-risk susceptibility loci and 
whether there were any differences in the strength of the associations. We found no differences, although there were some DCIS-specific loci that did not reach genome-wide significance. Therefore, our data largely support the theory that DCIS and IDC are, in fact, a continuum of the same disease, but we cannot exclude the possibility that there may be some low-risk susceptibility loci that have a strong association with stage 0 and a weaker association with stage I breast cancer. Identifying any such loci is important as it would identify a subset of DCIS that has a low risk of progression.

\section{Abbreviations}

DCIS, ductal carcinoma in situ; IDC, invasive ductal carcinoma; SNP, single nucleotide polymorphism

\section{Funding}

The authors have no sources of funding to declare.

\section{Authors' contributions}

VS and SAN made equal contributions to the conception and design of the letter, drafting the letter, and revising it critically for important intellectual content. Both authors read and approved the final manuscript.

\section{Competing interests}

The authors have no competing interests to declare.

Received: 28 April 2016 Accepted: 29 April 2016

Published online: 10 June 2016

\section{References}

1. Petridis C, Brook MN, Shah V, Kohut K, Gorman P, Caneppele M, et al. Genetic predisposition to ductal carcinoma in situ of the breast. Breast Cancer Res. 2016;18(1):22.

2. Michailidou K, Hall P, Gonzalez-Neira A, Ghoussaini M, Dennis J, Milne RC, et al. Large-scale genotyping identifies 41 new loci associated with breast cancer risk. Nat Genet. 2013;45(4):353-61.

3. Milne RL, Burwinkel B, Michailidou K, Arias-Perez J, Zamora MP, MenendezRodriquez $\mathrm{P}$, et al. Common non-synonymous SNPs associated with breast cancer susceptibility: findings from the Breast Cancer Association Consortium. Hum Mol Genet. 2014;23(22):6096-111.

4. Sopik V. Nofechs-Mozes S, Sun P. Narod SA. The relationship between loca recurrence and death in early-stage breast cancer. Breast Cancer Res Treat. 2016;155(1):175-85.

5. Narod S. Breast cancer: The importance of overdiagnosis in breast-cancer screening. Nat Rev Clin Oncol. 2016;13(1):5-6. 